\title{
Combined Endoscopic and Radiographic Evaluation of the Esophagus Immediately After Pneumatic Dilatation for Achalasia
}

\author{
Robert Bechara ${ }^{1 *}$, Paul Kortan², Gabor Kandel², Gary May², Ingrid Schwartz ${ }^{2}$, Norman Marcon² \\ ${ }^{1}$ Gastrointestinal Diseases Research Unit, Queen University Kingston General Hospital, 76 Stuart St. Kingston, Ontario, Canada, K7L 2V7 \\ ${ }^{2}$ Division of Gastroenterology, St Michael's Hospital, University of Toronto, 30 Bond St Toronto, ON M5B 1W8
}

Received: July 22, 2016; Accepted: September 20, 2016; Published: October 4, 2016

*Corresponding author: Robert Bechara, Gastrointestinal Diseases Research Unit, Queen University Kingston General Hospital, 76 Stuart St. Kingston, Ontario, Canada, K7L 2V7, Tel no: 6134498204; Email: bechara.robert@gmail.com

\begin{abstract}
Background and Aim: Current guidelines recommend that all patients undergoing pneumatic dilation have an esophagram within 24 hours of the procedure. Initially with a water soluble agent and then if negative using barium. Barium is denser and believed to adhere better to the mucosal wall allowing better delineation of the anatomy and as a result, is theoretically more sensitive for detection of perforations. However, the literature supporting this stems from case series of esophageal perforations from all aetiologies that have seldom included pneumatic dilation.
\end{abstract}

Methods: Here we describe our experience in over 200 pneumatic dilations using endoscopic examination and administration of water soluble contrast immediately post-dilation as the primary imaging modality after the pneumatic dilation to rule out perforation.

Results: 207 patients ranging in age from 20-87 years (mean 54 years) received pneumatic dilatation for achalasia. Ninety-seven $(47 \%)$ had no previous treatment, $110(53 \%)$ received at least one previous treatment. Of the 207 pneumatic dilations 3 perforations (1.5\%) occurred. Two of the three perforations were detected immediately on the combined endoscopic and radiologic examination.

Conclusion: Immediate endoscopic administration of lowosmolality water-soluble contrast has multiple advantages that include; minimizing unnecessary post-procedure imaging and allowing for the rapid diagnosis of perforation, resulting in the earliest opportunity for intervention.

\section{Introduction}

Achalasia is an esophageal motor disorder resulting from inhibitory neuron dysfunction causing loss of peristalsis and impaired lower esophageal sphincter relaxation. This leads to impaired food bolus propulsion and stasis in the esophagus. Patients can experience dysphagia, regurgitation, chest pain, weight loss and heartburn. Current treatment options include laparoscopic Hellermyotomy (LHM), pneumatic dilation, peroral endoscopic myotomy (POEM) and botulinum injection. A recent study found that rates of therapeutic success with pneumatic dilation were similar to Hellermyotomy. Pneumatic dilation is often the initial treatment of choice for eligible patients. It is effective and generally safe in experienced hands with an average perforation rate of approximately 4\% [1]. Esophageal perforation can result in pain, vomiting, dyspnea, fever, tachycardia, tachypnea, crepitus, and shock. However, the symptoms may be subtle and vague $[2,3]$. It has been repeatedly demonstrated that delayed diagnosis of esophageal perforation greater than 24 hours results in significantly increased mortality with rates up to $40 \%$ [4-6]. With early recognition and appropriate treatment, mortality can be decreased by up to $70 \%$ [6].

Current guidelines recommend that all patients undergoing pneumatic dilation have an esophagram within 24 hours of the procedure [7]. The esophagram should first be performed with a water soluble contrast agent. If a large perforation is present the water soluble contrast is rapidly reabsorbed and is less noxious to the media stinum than barium. If the initial esophagram is negative then a barium study is performed. Barium is denser and believed to adhere better to the mucosal wall allowing better delineation of the anatomy and as a result, is theoretically more sensitive for detection of perforations. However, the literature supporting this stems from case series of esophageal perforations from all aetiologies that have seldom included pneumatic dilation. Miss rates of esophageal perforation with water soluble contrast esophagrams have been reported to range from $0-50 \%$ [8-11]. The "gold standard" in these series varied from barium esophagrams, computed tomography (CT) and esophagogastroscopy.Nonetheless, barium esophagram have also been reported to miss rates of up to $10 \%$ [12].

Most water soluble contrast media are hypertonic solutions that can potentially cause severe pulmonary edema if aspirated. The risk of this adverse event is lessened by the use of lowosmolality water soluble contrast agents such as Omnipaque (iohexol).Low-osmolality water soluble agents are well tolerated 
by the lungs following aspiration with minimal histological reaction [13-15]. The literature pertaining to perforation after pneumatic dilation is sparse and the techniques for esophagram have only included contrast swallowing or contrast administered via a naso-esophageal tube. Here we describe our experience using endoscopic administration of water soluble contrast as the primary imaging modality after the pneumatic dilation (Figure 1).

\section{Methods}

Between January 1, 2006 and August 30, 2013, a total of 207 patients (93 women, 114 men) ranging in age from 20-87 years (mean 54 years) received pneumatic dilatation for achalasia. Written informed consent for pneumatic dilation was obtained from all patients. In this series, the data was prospectively collected and retrospectively examined.Ninety-seven (47\%) had no previous treatment, 110 (53\%) received at least one previous treatment (93 pneumatic dilation, 10 savary dilation, 10 LHM and 6 Botulinum injection). For all initial dilations a $30 \mathrm{~mm}$ Rigiflex Boston Scientific balloon was used. In patients receiving repeat dilations a $35 \mathrm{~mm}$ balloon was used. For all dilations the balloon was positioned at the gastroesophageal junction and inflated to 12 PSI for 30 seconds, deflated then inflated again to 12PSI for another 30 seconds (Figure 1).After dilation the bed is positioned in reverse trendelenburg at $\sim 45^{\circ}$, to prevent aspiration, the gastroscope advanced to the gastroesophageal junction, where a detailed endoscopic exam was performed. Omnipaque is injected endoscopically to obtain the esophagram. Subsequently, the patient does not undergo further radiologic testing if they are clinically asymptomatic. To our knowledge, this is the only series reporting on this method of examination immediately after

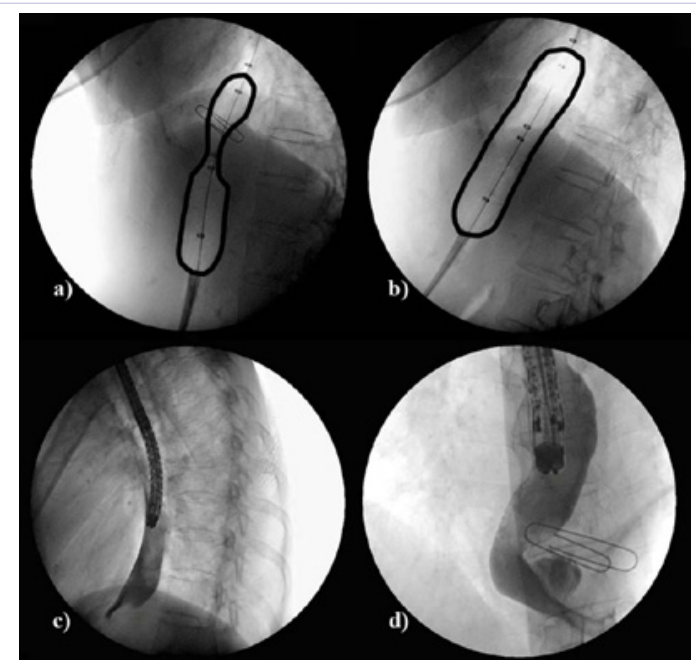

Figure 1: Pneumatic dilation with immediate post-procedure esophagram with endoscopically administered contrast

a) Balloon placement and initial inflation demonstrating waist.

b) Waist obliteration with dilation. Esophagrams of two different patients immediately post dilation

c) Demonstrating no perforation

D) Free perforation with contrast extravasation. pneumatic dilation using combined endoscopic and fluoroscopic techniques.

\section{Results}

Of the 207 pneumatic dilations 3 perforations (1.5\%) occurred. Two of the three perforations were detected immediately on the combined endoscopic and radiologic examination (figure 1) and both required further intervention. One patient received surgical repair with Heller myotomy and was discharged in 7 days, the other patient received a covered stent placed immediately after contrast exam, was clinically asymptomatic and was sent back to the referring facility without any adverse events. The one perforation that was not detected on immediate post-dilation exam was detected on CT performed due to ongoing severe chest pain in the recovery room. The CT revealed no extravasation but a small amount of intramural air and contrast and was managed conservatively. Of the 207 dilations post-procedure CT was performed on 9 patients, two of which had perforations. No cases of aspiration, pulmonary edema, or deaths occurred as a result of the immediate post-procedure esophagram with endoscopically administered contrast.

\section{Conclusion}

We propose that immediate endoscopic administration of low-osmolality water-soluble contrast has multiple advantages. This technique allows for the quickest possible diagnosis of perforation, resulting in the earliest opportunity for intervention, decreasing morbidity and mortality. The endoscopic examination of the esophagus potentially increases the sensitivity of detecting perforation as it has also been used as a primary modality to detect perforation [16]. The contrast can be injected multiple times in a targeted manner to closely scrutinize suspect areas, which is not possible with orally or tube-administered contrast. It is cost effective, as the patients do not require further radiologic examination post-procedure. Low-osmolality water soluble contrast results in minimal pulmonary adverse events if aspirated. This technique can also be applied POEM, a more recent treatment introduced for achalasia. In addition, it does not result in significant artifact if a subsequent CT examination is required. Limitations of this study are that the data was retrospectively analyzed which is subject to bias. In addition, three perforations occurred, thus only three primary endpoints were obtained. Ideally, a multicenter prospectively study should be performed in order to maximize the number of primary endpoints (perforations)leading to a more accurate representation of the operating characteristics of the combined immediate endoscopic administration of low-osmolality water soluble contrast as a method for perforation detection after pneumatic dilation.

\section{References}

1. Boeckxstaens GE, Annese V, des Varannes SB, Stanislas Chaussade, Mario Costantini, Antonello Cuttitta, et al. Pneumatic dilation versus laparoscopic Heller's myotomy for idiopathic achalasia. N Engl J Med. 2011;364:1807-1816. DOI: 10.1056/NEJMoa1010502.

2. Wu JT, Mattox KL, Wall MJ Jr. Esophageal perforations: new perspectives and treatment paradigms. J Trauma. 2007;63(5):11731184. 
3. Soreide JA, Konradsson A, Sandvik OM, Øvrebø K, Viste A. Esophageal perforation: clinical patterns and outcomes from a patient cohort of Western norway. Dig Surg. 2012;29(6):494-502. doi: $10.1159 / 000346479$.

4. Shaker H, Elsayed H, Whittle I, Hussein S, Shackcloth M. The influence of the 'golden 24-h rule' on the prognosis of oesophageal perforation in the modern era. Eur J Cardiothorac Surg. 2010;38(2):216-222. doi: 10.1016/j.ejcts.2010.01.030

5. Soreide JA, Viste A. Esophageal perforation: diagnostic work-up and clinical decision-making in the first 24 hours. Scand J Trauma Resusc Emerg Med. 2011;19:66. doi: 10.1186/1757-7241-19-66

6. Brinster CJ, Singhal S, Lee L, Marshall MB, Kaiser LR, Kucharczuk JC Evolving options in the management of esophageal perforation. Ann Thorac Surg. 2004;77(4):1475-1483

7. Vaezi MF, Pandolfino JE, Vela MF. ACG clinical guideline: diagnosis and management of achalasia. Am J Gastroenterol. 2013;108:12381249. doi:10.1038/ajg.2013.196.

8. Buecker A, Wein BB, Neuerburg JM, R W Guenther. Esophagea perforation: comparison of use of aqueous and barium-containing contrast media. Radiology. 1997;202(3):683-686. DOI: 10.1148/ radiology.202.3.9051016.

9. Swanson JO, Levine MS, Redfern RO, Rubesin SE. Usefulness of high density barium for detection of leaks after esophagogastrectomy, total gastrectomy, and total laryngectomy. AJR Am J Roentgenol 2003;181(2):415-420.

10. Sacks TB. Oral contrast material for fluoroscopic diagnosis of esophageal rupture. AJR Am J Roentgenol. 1995;164(2):512.

11. Brick SH, Caroline DF, Lev-Toaff AS, Friedman AC, Grumbach K, Radecki PD. Esophageal disruption: evaluation with iohexol esophagography. Radiology. 1988;169(1):141-143.

12. White CS, Templeton PA, Attar S. Esophageal perforation: CT findings. AJR Am J Roentgenol. 1993;160(4):767-770.

13. Ginai AZ, ten Kate FJ, ten Berg RG, Hoornstra K. Experimental evaluation of various available contrast agents for use in the upper gastrointestinal tract in case of suspected leakage. Effects on lungs. $\mathrm{Br}$ J Radiol. 1984;57(682):895-901.

14. Harris JA, Bartelt D, Campion M, Gayler BW, Jones B, Hayes A, et al. The Use of Low-Osmolar Water-Soluble Contrast in Videofluoroscopic Swallowing Exams. Dysphagia. 2013;28(4):520-527. doi: 10.1007/ s00455-013-9462-0.

15. Morcos SK. Review article: Effects of radiographic contrast media on the lung. Br J Radiol. 2003;76(905):290-295.

16. Hogan BA, Winter DC, Broe D, Broe P, Lee MJ. Prospective trial comparing contrast swallow, computed tomography and endoscopy to identify anastomotic leak following oesophagogastric surgery. Surg Endosc 2008;22(3):767-771. 